\title{
Descripción parcial y montaje de un esqueleto de tortuga negra Chelonia mydas agassizii (Bocourt, 1868)
}

\author{
Partial description and assemblage of a black turtle \\ Chelonia mydas agassizii (Bocourt, 1868) skeleton
}

Benjamín Cáceres Murrie ${ }^{1,2}$, Anelio Aguayo Lobo ${ }^{1,3}$, Nicolás Muñoz García ${ }^{1}$, Miguel Cáceres Murrie ${ }^{1} \&$ Lautaro Oyarzún Galaz ${ }^{4}$

Chelonia mydas (Linnaeus, 1758) es una especie de tortuga marina, omnivora, distribuida en aguas tropicales y templadas de los océanos Atlántico, Índico y Pacífico (Donoso-Barros, 1966; Cardona et al. 2009). La historia de vida de esta especie es compleja, debido a las escalas de espacio y tiempo involucradas, recordando que son animales que habitan el planeta Tierra durante millones de años hasta la actualidad. Actualmente, se discute sobre su estado taxonómico en la cuenca del Pacífico. La literatura muestra diversas evidencias respecto a si las formas representan subespecies o especies distintas (Karl \& Bowen, 1999; Pritchard, 1999). Sin embargo, investigaciones recientes han demostrado que la población del Pacífico Oriental antiguamente denominada tortuga negra Chelonia mydas agassizii (Bocourt, 1868), es sólo una forma melanística del género Chelonia; la cual se distingue por su color verde oscuro, su forma convexa y su caparazón angosto (Dutton et al. 2014, 2019).

En el Pacífico Oriental, esta tortuga se distribuye desde Baja California, México, hasta Perú, anidando especialmente en las costas de Michoacán e islas Revillagigedo, México, en la costa Pacífica de Costa Rica y en las islas Galápagos, Ecuador (Márquez, 1990). Sin embargo, individuos vagabundos han sido registrados hasta la Columbia Británica, Canadá, por el norte, y a lo largo de toda la costa de Chile, por el sur, incluyendo los dos registros más australes del Pacífico Oriental, en la boca occidental del Estrecho de Magallanes (Guzmán \& Campodónico, 1973; Cáceres et al. 2018).

Desde el año 2014, el Museo de Historia Natural Río Seco (MHNRS) en la ciudad de Punta
Arenas, cumple con la misión de revalorizar y poner en valor el patrimonio natural de la región de Magallanes. Actualmente, cuenta con una colección de alrededor de 300 piezas osteológicas, las cuales se encuentran disponibles para la investigación, educación y exhibición. El objetivo de este trabajo es describir parcialmente el esqueleto del segundo ejemplar registrado, el cual fue ingresado a la colección de zoología del MHNRS para, posteriormente, ser exhibido a la comunidad.

El esqueleto del ejemplar fue preparado y posteriormente fue articulado y montado para su exhibición por dos investigadores del Museo (M.C.M. \& B.C.M.), utilizando la técnica de la adaptación en osteotecnia. Dicho proceso corresponde al conjunto de procedimientos naturales, químicos $y / o$ físicos a través de los cuales se puede tratar un espécimen con el fin de obtener un hueso, segmentos o esqueletos limpios, sin presencia de residuos orgánicos, facilitando la observación de formaciones anatómicas para su uso en docencia e investigación (RodríguezPalomo \& Ramírez, 2009; Villarroel \& Troncoso,

\footnotetext{
1 Asociación de Investigadores del Museo de Historia Natural Río Seco, Punta Arenas.

$\triangle$ benjamíncaceresm@gmail.com

2 Centro de Estudios del Cuaternario Fuego Patagonia y Antártica (CEQUA), Punta Arenas.

3 Instituto Antártico Chileno (INACH), Punta Arenas.

4 Facultad de Ciencias del Mar y de Recursos Naturales, Universidad de Valparaíso, Valparaíso.
} 
2017). Cabe señalar, sin embargo, que la técnica desarrollada por el MHNRS para la conservación de esqueletos, se distingue de esta definición general, dado que algunos tejidos orgánicos como cartílagos y ligamentos, presentes en las articulaciones y dependiendo de la singularidad de la pieza (cuerpo y forma) tratada, no son eliminados por completo, ya que sirven de articuladores naturales y ayudan a dar una mayor plasticidad en virtud de la presentación de los modelos.

El cadáver, una vez trasladado a las dependencias del MHNRS, fue lavado cuidadosamente con agua fría y revisado externamente en su posición dorsal y ventral para identificar traumas, anormalidades, heridas, deformidades o extremidades faltantes. Dado el estado avanzado de descomposición en el cual se encontró el ejemplar, el cráneo estaba desprovisto de su mandíbula, huesos premaxilares y aparato hioideo, mientras que la extremidad posterior derecha se encontraba ausente. De la columna vertebral faltaban algunas de las últimas vértebras caudales, imposibilitando la determinación de sexo. El ejemplar fue donado para la colección de zoología del MHNRS, con el objeto de preparar el esqueleto para su posterior estudio y exhibición en la muestra permanente de dicha institución. La nomenclatura del sistema óseo empleado en este trabajo es siguiendo a Wyneken (2001).

El proceso de limpieza del esqueleto (Fig. 1a) de la tortuga negra, se llevó a cabo removiendo cuidadosamente la mayor cantidad de tejidos blandos posible, incluyendo musculatura y órganos principalmente y desarticulando las extremidades, todo con la ayuda de bisturís y pinzas. Paralelamente, el esqueleto iba siendo rociado con agua recién hervida, hasta que hacia el final de la jornada, el mismo quedara sumergido en agua caliente con $20 \mathrm{ml}$ de detergente durante 24 horas. Al día siguiente, el esqueleto se enjuagó en agua fría, para luego continuar con la labor de descarnado, preocupándose de no desarticular o separar los huesos que componen las extremidades. Una vez finalizada la etapa de descarnado, el esqueleto fue sumergido en agua caliente con $10 \mathrm{ml}$ de cloro durante 24 horas. $\mathrm{Al}$ día siguiente, el esqueleto fue enjuagado en agua fría y se descarnaron los últimos restos de tejido para luego ser sumergido en una solución de agua oxigenada al 30\% durante 24 horas, cuya acción permite blanquear los restos óseos y detener la descomposición de los escasos tejidos blandos. Una vez que el esqueleto fue retirado y enjuagado nuevamente, se fijó con palillos de bambú en una plancha de plumavit con el objetivo de secar los huesos durante aproximadamente 48 horas en la posición elegida para su posterior montaje (Fig. 1b). El esqueleto se articuló con la ayuda de ensambles de alambre de acero inoxidable y pegamento Loctite 401, para luego ser exhibido en su montaje final. Por último, se confeccionó una caja metálica forrada interiormente con espejos y dos fierros que permiten darle sujeción a la pieza dentro de la misma. Los espejos, además de cerrar el montaje en un aparato estético (Déotte, 2012), permiten una aproximación al esqueleto desde todos los ángulos posibles por parte del espectador (Fig. 2).

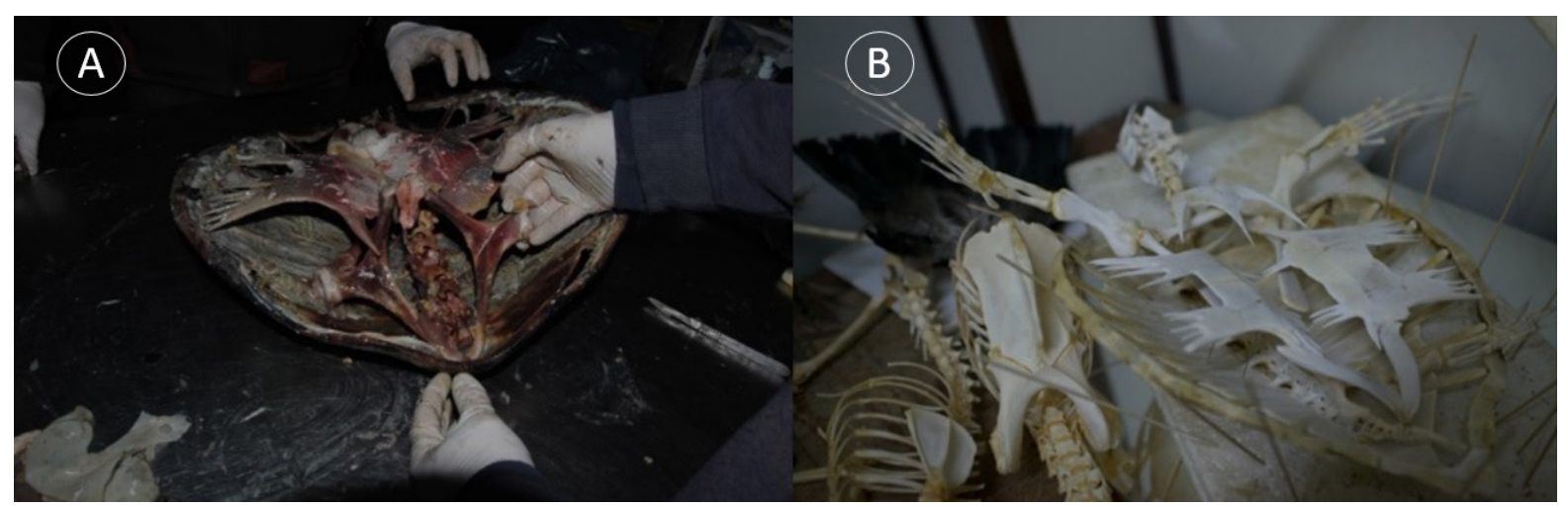

Fig. 1. a) Esqueleto en proceso de limpieza. b) Ejemplar articulado y en proceso de secado. 


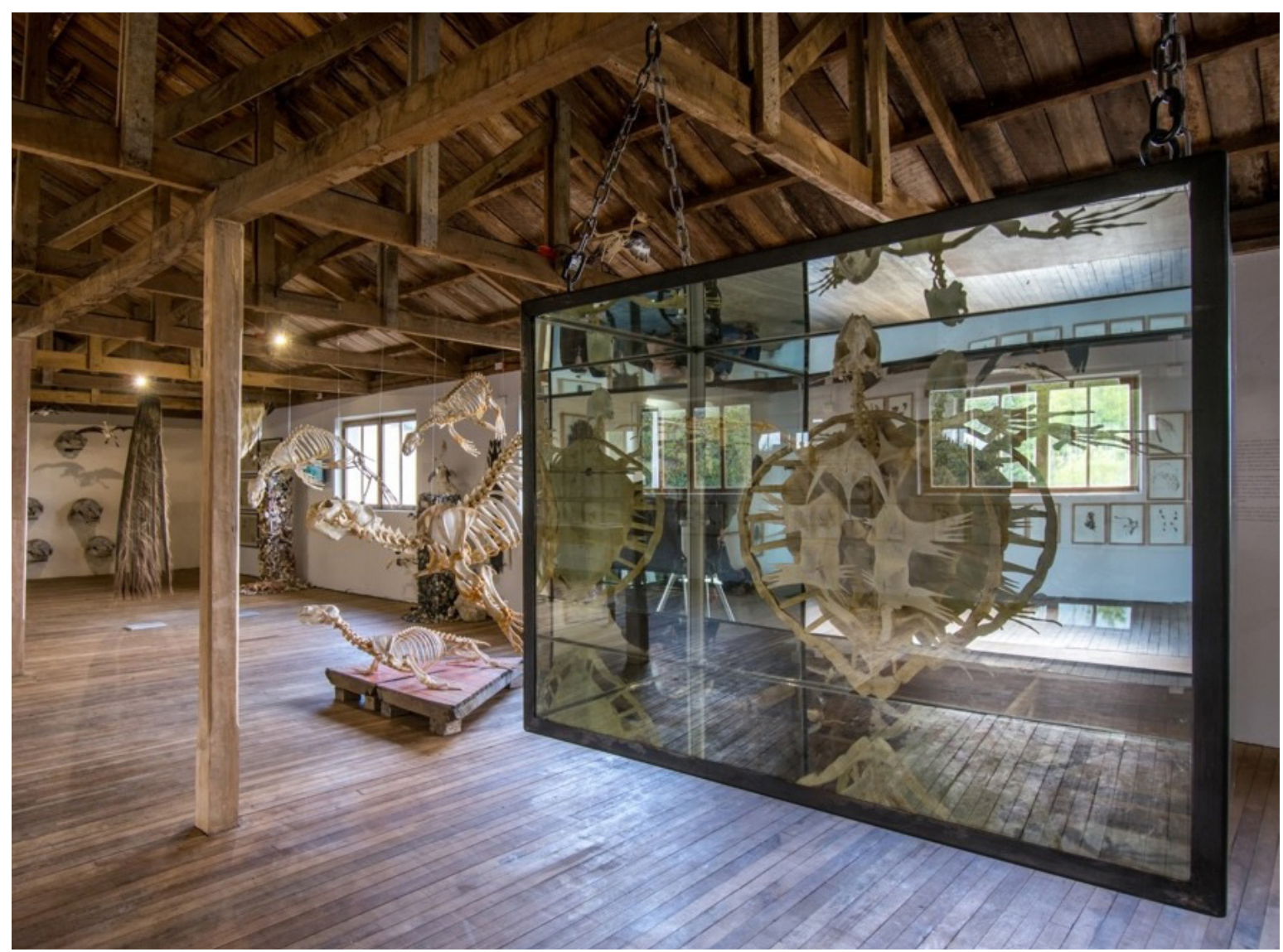

Fig. 2. Montaje final de la tortuga negra en las dependencias del Museo de Historia Natural Río Seco.

\section{DESCRIPCIÓN DEL ESQUELETO}

\section{Cráneo}

El cráneo comprende la caja, la mandíbula y el aparato hioideo. La caja craneana semeja un triángulo isósceles (Fig. 3a), que mide $124 \mathrm{~mm}$ de largo, sin considerar los premaxilares. El ancho del cráneo es de $79 \mathrm{~mm}$ de ancho. Su vértice está constituido por los premaxilares y los maxilares. En la línea media se encuentran los prefrontales, frontales, parietales y el supraoccipital (Fig. 3a), mientras que en su vista ventral (Fig. 3b), se distinguen el vómer, palatino, pterigoides, basiesfenoides y exoccipital. Lateralmente (Fig. $4 a$ y $4 b)$, la caja está constituida por los maxilares, yugales y postorbitales. La base de la caja (Fig. $5 a$ y 5b) está constituida por los escamosales, exoccipitales y supraoccipital. Este último elemento se prolonga hacia atrás notoriamente y una pequeña parte conforma el borde del foramen magnum del cráneo (Fig. 5b).

\section{Esqueleto axial}

El esqueleto axial está compuesto por la columna vertebral, el caparazón o espaldar y el plastrón o peto. La longitud total rectilínea (LTR) del caparazón es de $39.8 \mathrm{~cm}$, mientras que el ancho total rectilíneo (ATR) es de $47 \mathrm{~cm}$.

En su vista dorsal (Fig. 6a), se observan en el centro las placas neurales equivalentes al extremo de las apófisis vertebrales. En su extremo anterior, se encuentra la placa nucal, mientras que en el extremo posterior se distingue la placa pigial. Lateralmente, destacan las costillas, las placas costales y las placas marginales, delimitando el caparazón. En la vista ventral del esqueleto axial (Fig. 6b), se observan seis de las siete vértebras cervicales y algunos cuerpos 
vertebrales, a través de los huesos en proceso de osificación del plastrón o peto y algunos cuerpos vertebrales de las dorsales, lumbares, sacrales y caudales. Lamentablemente, estas últimas no venían completas, por tanto, no serán descritas. En el centro, se observa que el plastrón está compuesto por nueve huesos: dos epiplastrones anteriores y un entoplastron medio, el cual es un carácter diagnóstico de la especie (Wyneken, 2001), dos hyoplastrones antero-laterales, dos hypoplastrones postero-laterales y dos xiphiplastrones posteriores (Fig. 6b).

\section{Esqueleto apendicular}

El esqueleto apendicular está compuesto por sus extremidades anteriores y posteriores transformadas en aletas. Las primeras están constituidas por la cintura escapular, el húmero, radio, cúbito, huesos carpianos, metacarpianos y las falanges de los cinco dígitos, de los cuales el primero posee una garra. La cintura escapular está constituida por la escápula, el coracoides, el proceso acromial y la fosa glenoidea, la cual recibe la cabeza del húmero. La cintura pélvica está

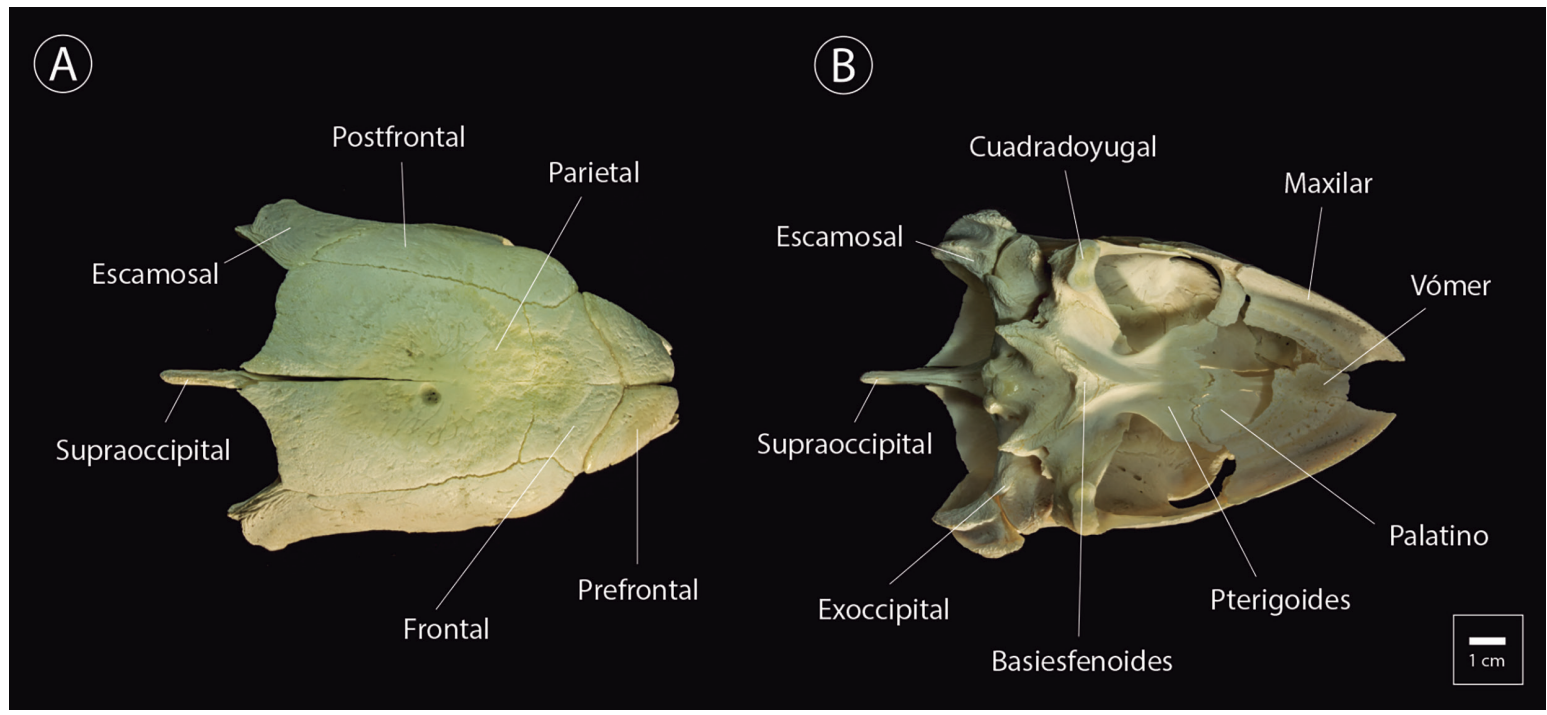

Fig. 3. Vistas del cráneo del ejemplar en estudio. a) Vista dorsal. b) Vista ventral.

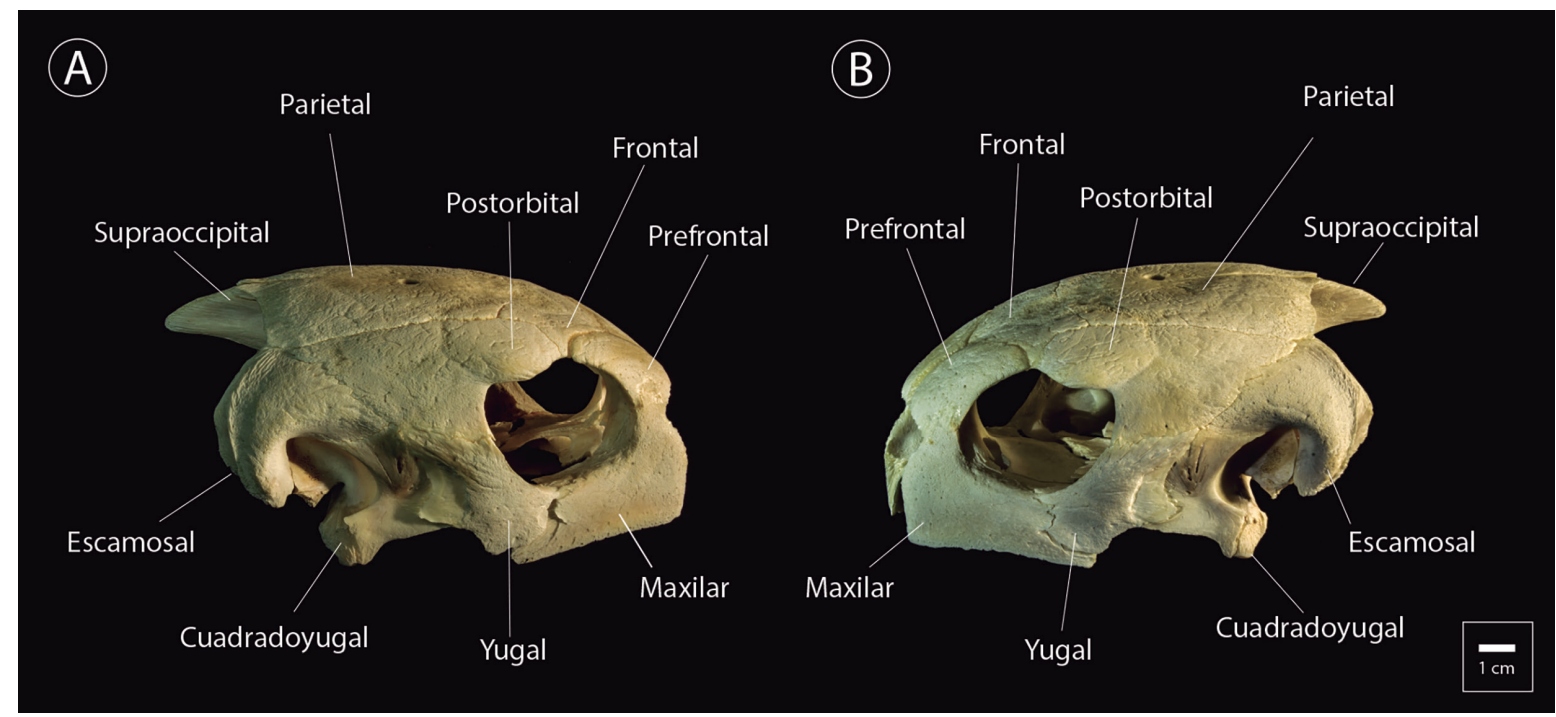

Fig. 4. Vista lateral derecha (a) y Vista lateral izquierda (b). 


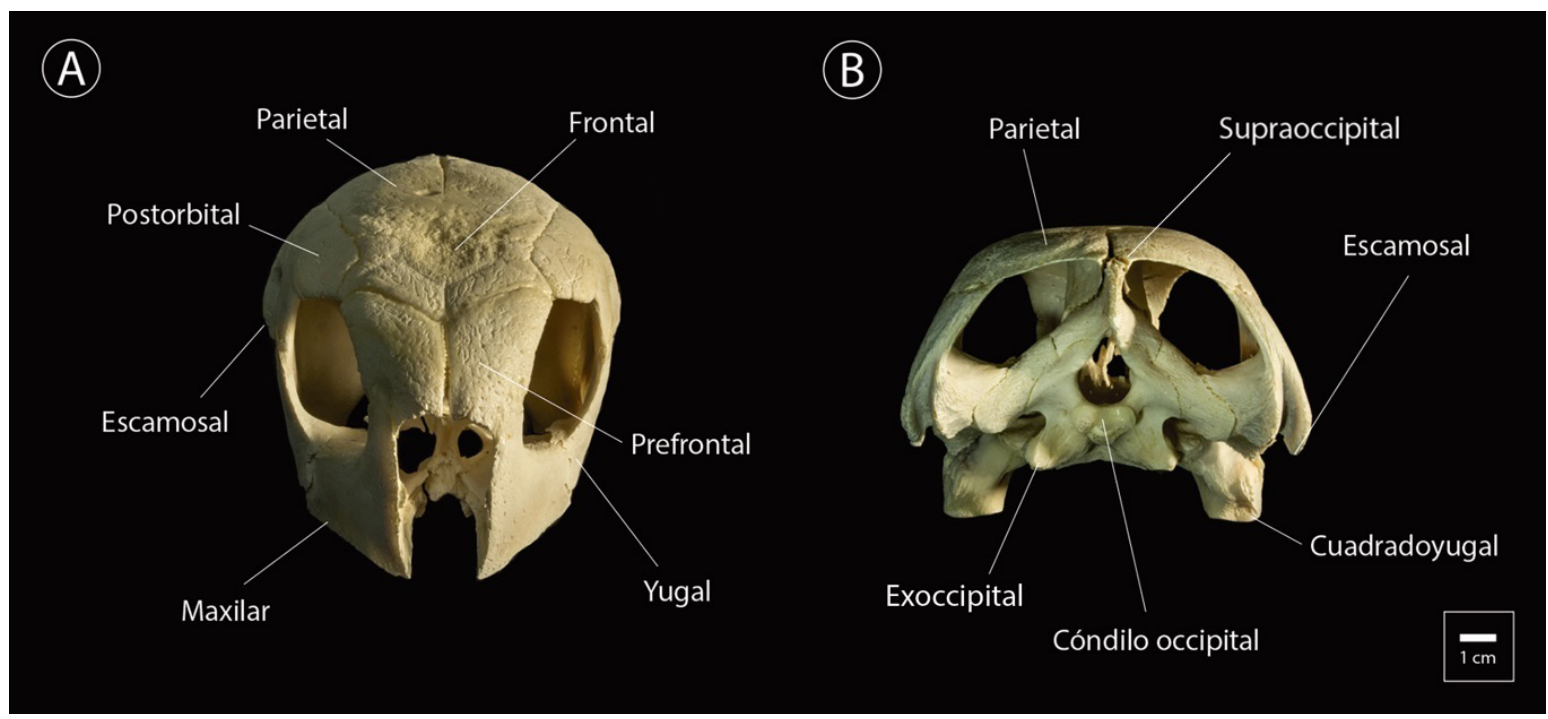

Fig. 5. Vista anterior (a) y Vista posterior (b).

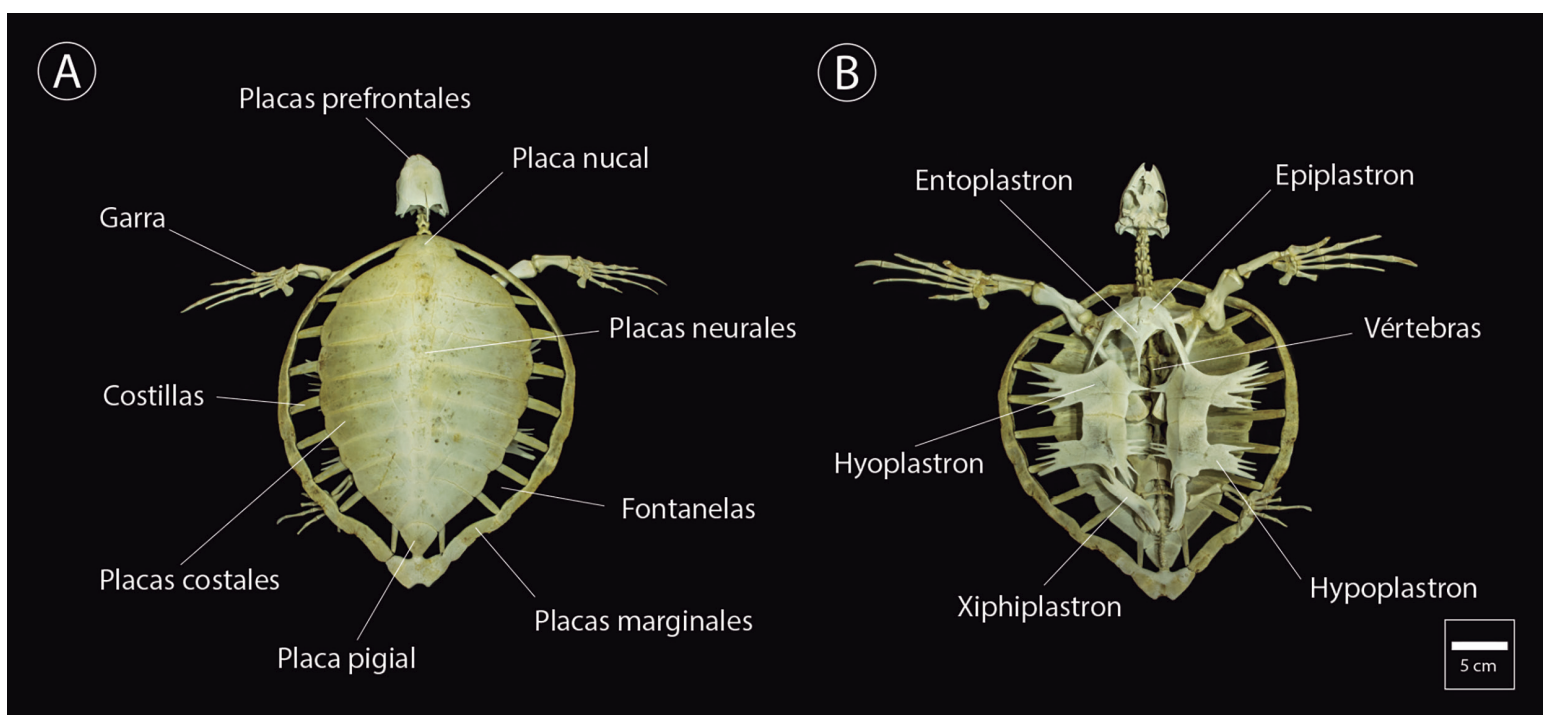

Fig. 6. Sistema esquelético del ejemplar en estudio. a) Vista dorsal. b) Vista ventral (Tomado de Cáceres et al. 2018).

constituida por tres huesos: púbis, isquion e ilion, notándose parcialmente en la Fig. 3b, debido a que se encuentran semi cubiertos por los hipoplastrones postero-laterales.

Los tres huesos de la pelvis forman una cavidad para recibir la cabeza del fémur denominada acetábulo, que en las tortugas jóvenes está constituida por cartílago y se osifica en los adultos. La extremidad posterior está conformada por el fémur, tibia y peroné, huesos tarsianos, metatarsianos y las falanges de los cinco dígitos. Tanto en la vista dorsal y ventral, las extremidades anteriores emergen casi completamente fuera del borde del caparazón (Fig. 6a), mientras que las extremidades posteriores apenas sobresalen fuera del espaldar, con sus falanges (Fig. 6b).

\section{CONCLUSIONES}

Debido al tamaño del cráneo, del caparazón y del plastrón, así como el grado de osificación y separación de los elementos óseos del plastrón y del tamaño de las fontanelas, se deduce que el animal era un juvenil de Chelonia mydas agassizii. 
El depósito de este ejemplar contribuye al conocimiento y valorización del patrimonio natural de la región de Magallanes, cuyas aguas forman parte del ecosistema subantártico, donde el esqueleto del ejemplar descrito pone en evidencia la complejidad de nuestros ecosistemas.

\section{AGRADECIMIENTOS}

Los autores desean agradecer al Sr. Erwin Soto por su valiosa donación para el Museo de Historia Natural Rio Seco. Al arqueólogo Sr. Alfredo Prieto y al biólogo Sr. Alejandro Kusch, por su colaboración e interés en la preparación del ejemplar. A los colegas del Museo de Historia Natural Río Seco, a la Srta. Gabriela Garrido, a la Srta. Aymara Zegers y al Sr. Sebastián Aspée, por la confección de la caja y posterior montaje y a la Srta. Antonia Ríos por el diseño de las figuras. Finalmente, los autores agradecen a las direcciones del Centro de Estudios del Cuaternario Fuego Patagonia y Antártica (CEQUA) y del Instituto Antártico Chileno (INACH), por las facilidades otorgadas para preparar la presente contribución.

\section{LITERATURA CITADA}

Cáceres, B., Aguayo Lobo A., Scholfield, D., Arcos F., Muñoz, N., Cabello, J., \& Acevedo, J. (2018). New record of black turtle Chelonia mydas agassizii in high latitudes of eastern South Pacific Ocean. Journal of Life Sciences, 12, 150-158

Cardona, L., Aguilar A., \& Pasos, L. (2009). Delayed ontogenic dietary shift and high levels of omnivory in green turtles (Chelonia mydas) from the NW coast of Africa. Mar Biol, 156, 1487-1495.

Carr, A. (1952). Handbook of turtles of the United States, Canada and Baja California. Ithaca: Cornstock Publish Associates, Cornell University Press

Déotte, J. - L. (2012). ¿Qué es un aparato estético? Santiago de Chile: Ediciones Metales Pesados.

Donoso-Barros, R. (1966). Reptiles de Chile. Santiago de Chile: Ediciones Universidad de Chile.
Dutton, P. H., Jensen, M. P., Frey, A., LaCasella, E., Balazs, G., Zárate, P., Chassin-Noria, O.,... \& Velez, E. (2014). Population structure and phylogeography reveal pathways of colonization by a migratory marine reptile (Chelonia mydas) in the central and eastern Pacific. Ecology and Evolution, 4(22),4317-4331.

Dutton P. H., Le Roux, R., La Casella E., Seminoff, J., Eguchi, T., \& Dutton, D. (2019). Genetic analysis and satellite tracking reveal origin of the green turtles in San Diego Bay. Marine Biology, 166, 3.

Formas, R. (1976). Encuentro de Chelonia mydas agassizii (Testudinata: Chelonidae) en la costa de Valdivia. Boletín de la Sociedad de Biología de Concepción, L, 213-214.

Guzmán, L., \& Campodónico, I. (1973). Presencia de Chelonia mydas agassizii Bocourt en Magallanes. Anales del Instituto de la Patagonia, 4, 339-341.

Karl, S. A., \& Bowen, B. W. (1999). Evolutionary significant units versus geopolitical taxonomy: molecular systematics of an endangered sea turtle (genus Chelonia). Conservation Biology, 13, 990-999.

Márquez, M., R. (1990). Sea turtles of the world. An annotated and illustrated catalogue of sea turtle species known to date. En R. Márquez. FAO species Catalogue. Vol. 11. Sea turtles of the world, An annotated and illustrated catalogue of sea turtle species known to date (81 p.). Ciudad de México: FAO Fisheries Synopsis.

Pritchard, P. (1999). Status of the Black Turtle. Conservation Biology, 13, 1000-1003.

Riquelme, L., Abarzúa, R., \& Leichtle, J. (2018). Adaptación de osteotecnia para el montaje de un ejemplar de delfín de Risso Gampus griseus (Cuvier, 1812). Revista Medicina Veterinaria Investigativa, 1(1), 102-108.

Rodríguez-Palomo, D., \& Ramírez Zamora, J. (2009). Técnica de conservación de huesos en peróxido de hidrógeno. Medicina Legal de Costa Rica, 26(2), 117-123.

Villarroel, G. M. A., \& Troncoso, F. (2017). Combinación de osteotecnia más conservación de músculos en montaje único 
de Canis lupus familiaris. International Journal of Morphology, 35(1), 351-356.

Wyneken, J. (2001). The Anatomy of Sea
Turtles. U.S. Department of Commerce NOAA Technical Memorandum NMFSSEFSC-470, 1-172 pp. 
